\title{
Producción científica sobre Fotografía en las revistas de Documentación (2000-2019)
}

\author{
Juan Miguel Sánchez-Vigil, Antonia Salvador-Benítez, María Olivera-Zaldua \\ Facultad de Ciencias de la Documentación, Universidad Complutense de Madrid. \\ * Correo-e: jmvigil@ucm.es | ORCID iD: https://orcid.org/0000-0002-4388-0250 \\ ** Correo-e: asalvado@ucm.es | ORCID iD: https://orcid.org/0000-0003-0087-5707 \\ *** Correo-e: molivera@ucm.es | ORCID iD: https://orcid.org/0000-0001-6335-9094
}

Recibido: 12-01-20; 2a versión: 18-03-20; Aceptado: 02-04-20; Publicado: 18-02-21

\begin{abstract}
Resumen: Los estudios científicos sobre Fotografía se difunden generalmente en las publicaciones de Comunicación, Arte e Historia. Sin embargo, las revistas de Documentación son también, hipotéticamente, fuente de información para la investigación en la materia, especialmente en lo que se refiere al tratamiento y análisis (conservación, catalogación, digitalización y difusión). Bibliotecas, archivos, museos, fundaciones, institutos y empresas conservan un importante patrimonio fotográfico susceptible de ser reutilizado en diversos usos y aplicaciones. Es objeto de este artículo analizar la producción científica sobre Fotografía en las principales revistas de Documentación en los últimos veinte años (2000-2019), con el fin de obtener información sobre autoría, contenidos y temáticas específicas. La investigación ha permitido conocer que los trabajos son escasos, resultando 146 artículos de 161 autores pertenecientes a 61 instituciones públicas y privadas, en los que se analizan contenidos centrados en dieciséis materias generales. Se observa, como principales conclusiones, la necesidad de profundizar en la investigación sobre Fotografía desde el punto de vista documental, y prestar una mayor atención por parte de los editores a los descriptores o palabras clave de los artículos, con el fin de una mayor y mejor recuperación de la información.
\end{abstract}

Palabras clave: documentación fotográfica; fotografía; producción científica; revistas de documentación; investigación en documentación.

\section{Scientific Production regarding Photography in Documentation Journals (2000-2019)}

Abstract: Scientific studies on Photography are generally published in publications about Communications, Art and History. However, Journals in Documentation Science could be also, hypothetically, a source of information about subject, especially in regard to processing and analysis (conservation, cataloguing, digitalization and circulation). Libraries, archives, museums, foundations, institutes and companies possess important photographic collections that can be reused for a variety of purposes and applications. The aim of this article is to analyze the scientific production on Photography in the main Documentation Journals over the past twenty years (2000-2019), in order to obtain information on authorship, contents and specific subject matters. This research has allowed us to discover that very few studies have been conducted on this subject: 146 articles from 161 authors, belonging to 61 public and private institutions, which analyze contents focusing on sixteen general subjects. The most relevant conclusions are the need for a broader investigation on Photography from the documentary point of view, and for the publishers to pay greater attention to the descriptors or key words in the articles, in order to facilitating and improving the location of the information.

Keywords: photographic documentation; photography; scientific production; documentation journals; research on documentation

Cómo citar este artículo/Citation: Sánchez-Vigil, J. M.; Salvador-Benítez, A., Olivera-Zaldua, M. (2021). Producción científica sobre Fotografía en las revistas de Documentación (2000-2019). Revista Española de Documentación Científica, 44 (1), e287. https://doi.org/ 10.3989/redc.2021.1.1749

Copyright: (c) 2021 CSIC. Este es un artículo de acceso abierto bajo los términos de la licencia de uso y distribución Creative Commons Reconocimiento 4.0 Internacional (CC BY 4.0). 


\section{INTRODUCCIÓN}

Los estudios científicos sobre Fotografía generalmente se publican en revistas de Comunicación y Arte, mientras que se desconocen las investigaciones en el campo de la Documentación (bibliotecas, archivos, museos y departamentos de documentación de empresas públicas y privadas), relacionados con la conservación, el tratamiento y análisis de contenidos, la digitalización y la difusión de los mismos. La Documentación Fotográfica ha sido tradicionalmente impartida dentro de las asignaturas de Documentación Audiovisual desde la puesta en marcha de las Facultades de Ciencias de la Información, si bien adquirió entidad propia al crearse la Escuela de Biblioteconomía y Documentación de la Universidad Complutense de Madrid y, posteriormente, las Facultades de Documentación en ésta y en otras instituciones académicas.

Es objeto de este trabajo analizar la producción en las principales revistas científicas de Documentación en relación a la Fotografía, en el periodo comprendido entre enero de 2000 y diciembre de 2019. Se considera fundamental recuperar estos trabajos realizados sobre esta materia, teniendo en cuenta la transversalidad de la fotografía y la ingente cantidad de documentos que se conservan en las instituciones públicas y privadas, de cuyo tratamiento deriva su recuperación, reutilización y difusión.

Por otra parte, es también objetivo fundamental responder a una de las líneas del Programa de actuación del Plan Nacional de Conservación del Patrimonio Fotográfico (Ministerio de Cultura, 2015: $3.5,129)$, donde se propone: "Fomentar la creación de líneas de ayuda y acuerdos de colaboración con universidades y otros organismos para el estímulo de la investigación en fotografía en sus diferentes vertientes: conservación, autores, historia, colecciones, etc.", así como a los propósitos de la recién creada Asociación Española para la Enseñanza de la Fotografía (Anef, 2018).

Partiendo de estas premisas, se ha llevado a cabo una revisión general del estado de la cuestión y de las fuentes. En relación con las revistas seleccionadas se han considerado las referencias generales de Guallar y otros (2017) en "Revistas científicas de información y documentación: Análisis temático y metodológico"; y de manera concreta los trabajos sobre la Revista Española de Documentación Científica (Ríos, 2001), Revista General de información y Documentación (López y otros, 2001), - Documentación de las Ciencias de la Información (Arquero y Río, 2002), y también estudios generales (Baladrón y Correyero, 2012). Sobre la producción en la disciplina Documentación se han obtenidos datos de Delgado (2001), Arquero (2002, 2003), Osca y Mateo (2003), Grupo Scimago (2005, 2006), Jiménez y otros (2006), Salvador (2007), Jiménez (2007) y Cerdán y otros (2015).

Sobre el impacto de la investigación en general se han consultado los artículos: "Los mil y un reflejos de las publicaciones en el laberinto de espejos de las nuevas métricas", de Gorraiz (2018) y "Cómo analizar el impacto de los datos de investigación con métricas: modelos y servicios", de Ferrer y otros (2016), y sobre investigación en materias específicas han sido de gran interés los trabajos sobre Comunicación por Caffarel y otros (2017), Publicidad por Baladrón y otros (2017); Documentación informativa por López y otros (2005), y Documentación periodística por Guallar (2003).

\section{METODOLOGÍA}

El análisis de las publicaciones y sus contenidos se ha realizado sobre doce revistas de Documentación (tabla I), tomando como referencia los índices de impacto y su posición desde el año 2000, elaborados por el grupo de investigación EC3 (Evaluación de la Ciencia y de la Comunicación Científica) de la Universidad de Granada, así como el sistema de información RESH (Revistas Españolas de Ciencias Sociales y Humanidades) que integra los indicadores de calidad para las publicaciones científicas españolas, desarrollados por los grupos EPUC (CCHS-CSIC) y el citado EC3.

El modelo seguido para la elaboración del artículo ha sido el trabajo previo titulado: "Producción científica sobre comunicación y medios en las revistas de documentación, 2000-2014" de Sánchez Vigil y otros (2015). Todas las publicaciones que se han analizado tienen una antigüedad superior a los veinte años en los que se enmarca la investigación, es decir que todas fueron creadas antes del 2000, con excepción de Ibersid que salió en 2007. En el caso de Métodos de Información, se han contemplado sus dos etapas: 2000-2002, editada por la Asociación Valenciana de Especialistas en Información, y 2010-2019.

Con el fin de obtener la máxima información se han revisado todos los artículos y sus correspondientes sumarios, accediendo desde las webs institucionales y desde el portal bibliográfico Dialnet cuando ha sido necesario. Las consultas específicas se han realizado en las colecciones de la Biblioteca de la Facultad de Ciencias de la Documentación de la UCM. En varias publicaciones de los primeros años (Anabad, BiD, Cuadernos de Documentación Multimedia, Item y Métodos de Información) ha sido necesaria la lectura de los textos para extraer las palabras clave. 
Tabla I. Revistas de Documentación analizadas (2000-2019)

\begin{tabular}{|c|c|c|c|}
\hline & Título & Editor & Fechas \\
\hline AN & Anales de Documentación & Universidad de Murcia & 1998-2019 \\
\hline BD & BiD. Textos Universitarios & $\begin{array}{l}\text { Facultat de BiD (Universidad de de Barcelona) } \\
\text { y Estudis de Ciencias de la Informació i de la } \\
\text { Comunicació (Universidad Oberta de Catalunya) }\end{array}$ & 1998-2019 \\
\hline BA & Boletín de Anabad & $\begin{array}{l}\text { Asociación de Archiveros, Bibliotecarios, Museólogos y } \\
\text { Documentalistas }\end{array}$ & $1950-2019$ \\
\hline$C D$ & Cuadernos de Documentación Multimedia & $\begin{array}{l}\text { Departamento de Biblioteconomía y Documentación } \\
\text { de la Universidad Complutense de Madrid }\end{array}$ & 1992-2019 \\
\hline $\mathrm{DC}$ & $\begin{array}{l}\text { Documentación de las Ciencias de la } \\
\text { Información }\end{array}$ & $\begin{array}{l}\text { Departamento de Biblioteconomía y Documentación } \\
\text { de la Universidad Complutense de Madrid }\end{array}$ & $1976-2019$ \\
\hline EP & El Profesional de la Información & El Profesional de la Información/ SCP & 1998-2019 \\
\hline IB & Ibersid & Universidad de Zaragoza & 2007-2019 \\
\hline IT & Item & Col.legi Oficial de Biblioteconomía y Documentación & 1987-2019 \\
\hline MI & Métodos de Información & $\begin{array}{l}\text { Colegio Oficial de Biblioteconomía y Documentación } \\
\text { (Valencia) }\end{array}$ & $\begin{array}{l}1998-2002 \\
2010-2019\end{array}$ \\
\hline RE & $\begin{array}{l}\text { Revista Española de Documentación } \\
\text { Científica }\end{array}$ & $\begin{array}{l}\text { Consejo Superior de Investigaciones Científicas/ } \\
\text { CINDOC }\end{array}$ & 1977-2019 \\
\hline RG & $\begin{array}{l}\text { Revista General de Información y } \\
\text { Documentación }\end{array}$ & $\begin{array}{l}\text { Departamento de Biblioteconomía y Documentación } \\
\text { de la Universidad Complutense de Madrid }\end{array}$ & 1992-2019 \\
\hline SC & Scire & Universidad de Zaragoza & 1995-2019 \\
\hline
\end{tabular}

En el análisis se ha detectado que varios buscadores no respondían a los términos prefijados, y que algunos artículos de diversas revistas no incluían palabras clave, e incluso que determinados términos que aparecían en los títulos y/o resúmenes no figuraban en las claves, por lo que ha sido necesaria una revisión completa de todas las revistas y todos los artículos para una completa extracción de datos. Para la recuperación de la información se ha elaborado una base de datos diseñada al efecto con los siguientes campos: Revista; Autor; Filiación; Artículo; Fecha; Volumen y número; Páginas; Medio tratado; Tema específico; Palabras clave, y Observaciones. Una vez procesada la información, valorados y analizados los contenidos, se han obtenido los resultados que se presentan acompañados de tablas, figuras y anexos.

\section{RESULTADOS}

Tras el análisis de las revistas se ha obtenido información sobre los autores y su filiación, los centros de investigación, los contenidos, el número de artículos, las temáticas abordadas y las palabras clave específicas. Se incluyen además datos sobre la producción de autores y centros extranjeros, así como las empresas e instituciones tratados en los contenidos.

\subsection{Producción global y artículos por revistas}

El número total de artículos en las revistas seleccionadas es de 6674 en el periodo estudiado, de
Ios que solo $146(2,18 \%)$ tratan sobre Fotografía, cantidad que es muy escasa. Se advierte un considerable aumento en 2011 debido a la convocatoria de un monográfico sobre el tema por El Profesional de la Información. De los textos, 41 corresponden a esta revista $(0,61 \%)$, que ocupa el primer lugar, y 24 a Documentación de las Ciencias de la Información $(0,35 \%)$, que se sitúa en segunda posición; es decir que en dos revistas se ha publicado el 44,52 de todos los textos. La tercera en número de artículos es Ibersid, con 15 títulos (0,22\%). Con más de 10 títulos figuran Scire y la Revista General de Información y Documentación, mientras que el resto está por debajo de esta cantidad (Tabla II, Figura 1).

Al establecer la relación entre el número de artículos por cada revista y el número de textos sobre Fotografía, los resultados son los siguientes: Documentación de las Ciencias de la Información es la primera, con 24 artículos sobre 278 $(8,63 \%)$, Métodos de Información con 7 artículos sobre $192(6,64 \%)$ e Ibersid con 15 sobre 293 $(5,11 \%)$, ésta última en un periodo más corto ya que fue creada en 2007, y por lo tanto proporcionalmente con mayor atención al tema. El resto, salvo Scire con 11 artículos sobre 365 (3.01\%) no alcanza el $3 \%$, y en dos casos el tema en cuestión apenas tiene espacio: BiD con 2 artículos sobre $436(0,45 \%)$ e Item con 3 sobre $265(1,13 \%)$ (Tabla III, Figura 2). 
Tabla II. Artículos por revistas (2010-2019).

\begin{tabular}{|c|c|c|c|c|c|c|c|c|c|c|c|c|c|}
\hline Año & AN & BA & BI & CD & DC & EP & IB & IT & MI & RE & RG & SC & Total \\
\hline 2000 & 0 & 0 & 0 & 0 & 1 & 1 & 0 & 0 & 0 & 0 & 0 & 0 & 2 \\
\hline 2001 & 0 & 0 & 0 & 0 & 2 & 2 & 0 & 0 & 0 & 1 & 0 & 0 & 5 \\
\hline 2002 & 1 & 0 & 0 & 1 & 0 & 0 & 0 & 1 & 0 & 0 & 0 & 1 & 3 \\
\hline 2003 & 0 & 0 & 0 & 0 & 1 & 1 & 0 & 1 & 0 & 0 & 0 & 0 & 3 \\
\hline 2004 & 0 & 0 & 0 & 0 & 0 & 0 & 0 & 0 & 0 & 0 & 0 & 0 & 0 \\
\hline 2005 & 0 & 1 & 0 & 0 & 1 & 0 & 0 & 0 & 0 & 0 & 0 & 2 & 4 \\
\hline 2006 & 0 & 0 & 0 & 0 & 1 & 0 & 0 & 0 & 0 & 0 & 0 & 1 & 2 \\
\hline 2007 & 0 & 1 & 0 & 0 & 0 & 1 & 3 & 0 & 0 & 0 & 0 & 1 & 6 \\
\hline 2008 & 0 & 0 & 0 & 0 & 0 & 1 & 1 & 0 & 0 & 0 & 0 & 0 & 2 \\
\hline 2009 & 0 & 1 & 0 & 1 & 0 & 3 & 2 & 0 & 0 & 0 & 0 & 0 & 9 \\
\hline 2010 & 0 & 0 & 0 & 0 & 2 & 4 & 1 & 0 & 0 & 0 & 1 & 2 & 10 \\
\hline 2011 & 1 & 1 & 1 & 0 & 3 & 12 & 0 & 0 & 0 & 2 & 0 & 0 & 20 \\
\hline 2012 & 1 & 0 & 0 & 0 & 2 & 1 & 0 & 0 & 0 & 0 & 0 & 1 & 5 \\
\hline 2013 & 0 & 0 & 0 & 2 & 4 & 1 & 2 & 0 & 0 & 0 & 0 & 0 & 9 \\
\hline 2014 & 1 & 0 & 0 & 0 & 1 & 4 & 1 & 0 & 6 & 1 & 2 & 0 & 16 \\
\hline 2015 & 1 & 1 & 0 & 0 & 1 & 1 & 2 & 0 & 0 & 1 & 2 & 0 & 9 \\
\hline 2016 & 1 & 1 & 0 & 1 & 1 & 0 & 2 & 0 & 0 & 2 & 3 & 3 & 14 \\
\hline 2017 & 0 & 2 & 0 & 0 & 3 & 4 & 1 & 0 & 1 & 1 & 0 & 0 & 12 \\
\hline 2018 & 1 & 1 & 1 & 0 & 0 & 2 & 0 & 1 & 0 & 1 & 1 & 0 & 8 \\
\hline 2019 & 0 & 0 & 0 & 0 & 1 & 3 & 0 & 0 & 0 & 0 & 3 & 0 & 7 \\
\hline Total & 8 & 9 & 2 & 5 & 24 & 41 & 15 & 3 & 7 & 9 & 12 & 11 & 146 \\
\hline
\end{tabular}

Tabla III. Artículos totales y artículos sobre fotografía.

\begin{tabular}{|c|c|c|c|}
\hline Revista & Art. Total & Art. Foto & $\%$ \\
\hline Anales de Documentación (AN) & 316 & 8 & 2,53 \\
\hline Boletín de Anabad (BA) & 709 & 9 & 1,26 \\
\hline $\mathrm{BiD}(\mathrm{BI})$ & 436 & 2 & 0,45 \\
\hline Cuadernos de Documentación Multimedia (CD) & 347 & 5 & 1,44 \\
\hline Documentación de las CC. de la Información (DC) & 278 & 24 & 8,63 \\
\hline El Profesional de la Información (EP) & 2364 & 41 & 1,73 \\
\hline Ibersid (IB) & 293 & 15 & 5,11 \\
\hline Item. Revista de biblioteconomia i documentació (IT) & 265 & 3 & 1,13 \\
\hline Métodos de Información (MI) & 192 & 7 & 6,64 \\
\hline Revista Española de Documentación Científica (RE) & 659 & 9 & 1,36 \\
\hline Revista General de Información y Documentación (RG) & 450 & 12 & 2,66 \\
\hline Scire (SC) & 365 & 11 & 3,01 \\
\hline Total & 6674 & 146 & 2,18 \\
\hline
\end{tabular}


Figura 1. Artículos publicados por año (20002019)

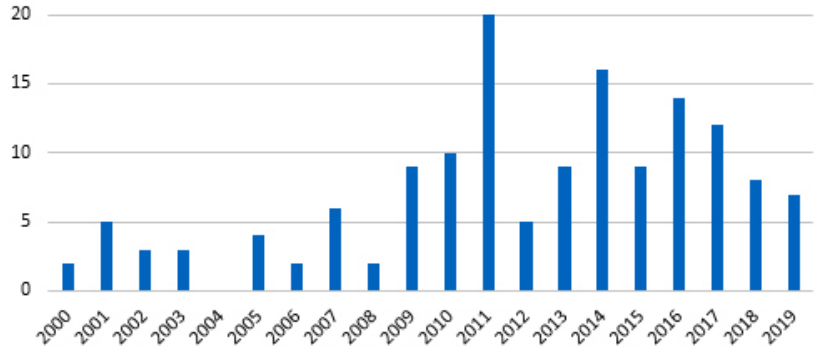

Figura 2. Porcentaje de artículos sobre fotografía en cada revista sobre el total publicado.

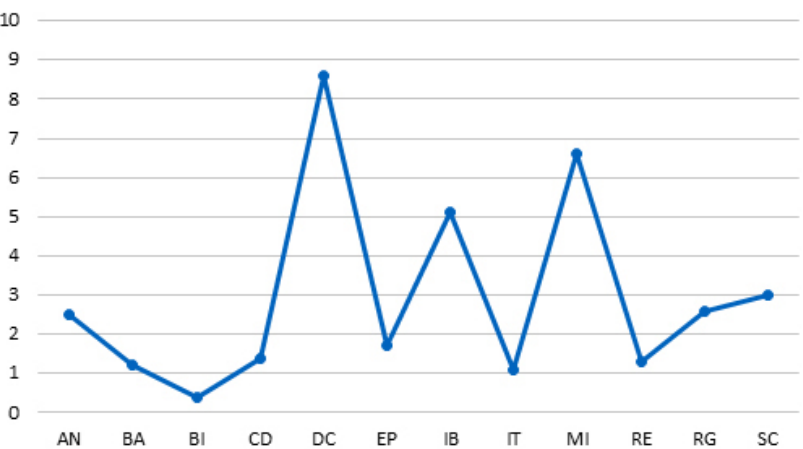

\subsection{Autoría y filiación a universidades, orga- nismos y empresas}

Los autores que escriben sobre la materia en las revistas seleccionados son 161 . El mayor número de trabajos es de Juan Miguel Sánchez Vigil con 36, seguido de María Olivera Zaldua con 25, Juan Carlos Marcos Recio con 15, Joaquín López del Ramo con 6, Jesús Robledano con 5, Antonia Salvador con 4, y Laia Fox, Lluis Codina y Antonio Ruiz con 3. El resto se encuentra por debajo de estas cantidades, sumando 45 autores con dos textos y 60 con tan solo uno (Tabla IV; Anexo 1).

Se han tenido en cuenta las coautorías de los textos, que presentan los siguientes datos: 45 artículos con dos autores, 38 con tres, 2 con cuatro y 1 con seis. De los autores citados más arriba con mayor número de artículos, la distribución es la siguiente:

- J. M. Sánchez Vigil: 10 como autor único, 10 con dos autores, y 16 con tres.

- M. Olivera Zaldua: 1 como autora única, 8 con dos autores, y 14 con tres.

- J. C. Marcos Recio: 1 con dos autores y 14 con tres.

- J. López del Ramo: 3 como autor único y 3 con dos autores.

- Jesús Robledano Arillo: 1 como autor único, 2 con dos autores y 2 con tres.

- A. Salvador Benítez: 1 como autora única, 1 con dos autores, y 2 con tres

- L. Fox Navarro: 1 como autora única y 2 con dos autores.

- LI. Codina: 2 como autor único y 1 con dos autores.

- A. Ruiz Rodríguez: 1 con dos autores, 1 con tres y 1 con cuatro.

En cuanto a la filiación de los investigadores, se ha realizado una división entre universidades (20 españolas y 11 extranjeras), instituciones públicas (14) y centros privados (16), a los que se suman dos profesionales (un abogado y un gestor de archivos) y cinco investigadores (un historiador, un sociólogo, un tecnólogo y dos documentalistas).

Tabla IV. Autores con mayor número de artículos.

\begin{tabular}{|c|l|l|c|}
\hline & Autor & Institución & Art. \\
\hline 1. & Sánchez Vigil, Juan Miguel & Universidad Complutense de Madrid & 36 \\
\hline 2. & Olivera Zaldua, María & Universidad Complutense de Madrid & 25 \\
\hline 3. & Marcos Recio, Juan Carlos & Universidad Complutense de Madrid & 15 \\
\hline 4. & López del Ramo, Joaquín & Universidad Rey Juan Carlos & 6 \\
\hline 5. & Robledano Arillo, Jesús & Universidad Carlos III & 5 \\
\hline 6. & Salvador Benítez, Antonia & Universidad Complutense de Madrid & 4 \\
\hline 7. & Fox Navarro, Laia & Institut d'Estudis Fotogràfics de Catalunya & 3 \\
\hline 8. & Codina, Lluis & Universidad Pompeu Fabra & 3 \\
\hline 9. & Ruiz Rodríguez, Antonio & Universidad de Granada & 3 \\
\hline
\end{tabular}


Tabla V. Universidades españolas. (AU: Autores; ART: Artículos)

\begin{tabular}{|l|c|c|l|c|c|}
\hline Universidad & Au & Art & Universidad & Au \\
\hline Complutense & 15 & 53 & Extremadura & 3 \\
\hline Granada & 11 & 7 & Europea de Madrid-CEES & 2 \\
\hline Carlos III & 7 & 8 & Las Palmas de Gran Canaria & 2 & 1 \\
\hline Barcelona & 6 & 4 & Jaime I (Castellón) & 2 \\
\hline Autón. de Barcelona & 5 & 3 & Rovira i Virgili (Tarragona) & 2 \\
\hline Pompeu Fabra & 5 & 6 & Málaga & 1 \\
\hline Rey Juan Carlos & 4 & 7 & Politécnica de Cartagena & 1 \\
\hline Murcia & 4 & 4 & Politécnica de Valencia & 1 \\
\hline Salamanca & 4 & 1 & Valladolid & 1 \\
\hline Zaragoza & 3 & 3 & Vigo & 1 \\
\hline
\end{tabular}

Tabla VI. Universidades extranjeras. (AU: Autores; ART: Artículos)

\begin{tabular}{|l|c|c|l|c|c|}
\hline Universidad & Au & Art & Universidad & Aurt \\
\hline $\begin{array}{l}\text { Estaduale Paulista Julio de } \\
\text { Mesquita Filho (Sao Paulo, Brasil) }\end{array}$ & 13 & 7 & Cauca (Colombia) & 1 \\
\hline Autónoma San Luis Potosí (México) & 2 & 1 & Federal de Alagoas (Brasil) & 1 \\
\hline $\begin{array}{l}\text { Central Marta Abreu de las Villas } \\
\text { (Cuba) }\end{array}$ & 2 & 1 & UNAM (México): & 1 \\
\hline $\begin{array}{l}\text { Instituto de la Cinematografía de } \\
\text { la Universidad de la República de } \\
\text { Uruguay }\end{array}$ & 2 & 1 & University of China & 1 \\
\hline $\begin{array}{l}\text { Benemérita Autónoma de Puebla } \\
\text { (México) }\end{array}$ & 1 & 3 & $\begin{array}{l}\text { University of Wisconsin-Parkside } \\
\text { (EE.UU) }\end{array}$ & 1 \\
\hline Cienfuegos (Cuba) & 1 & 1 & Total & 1 \\
\hline
\end{tabular}

Las universidades son las que mayor número de investigaciones generan, con un dato de gran interés en cuanto a la producción científica en centros españoles y extranjeros. Es significativo que el $35,5 \%$ de los textos procedan de profesorado de universidades de fuera del país, con gran diversidad: Colombia, Cuba, China, Estados Unidos, Uruguay, México y Brasil, que destaca con 13 autores (Tablas $\mathrm{V}$ y $\mathrm{VI}$ ).

\subsection{Impacto de la producción}

En cuanto al impacto de la producción científi$\mathrm{ca}$, se han recopilado las citas correspondientes a los artículos de los 10 autores con mayor número de publicaciones, debido a que la mayoría de los que figuran en el corpus (Anexo) tienen tan solo uno o dos artículos publicados. Las fuentes han sido Google Scholar y Research Gate, resultando un total de 217 citas de las que 95 corresponden a los trabajos de Sánchez Vigil, como autor único o en colaboración con los autores que figuran entre los que más han publicado (Marcos Recio, Olivera
Zaldua y Salvador Benítez): 29 citas como único, 21 con Olivera Zaldua, 5 con Fernández Fuentes y 4 con Salvador Benítez, más 34 con Olivera Zaldua y Marcos Recio, y 2 con Olivera Zaldua y Salvador Benítez. Se añaden a las 95 anteriores otras 2 de Juan Marcos en colaboración, y 3 de Olivera Zaldua como autora única. Del resto de autores, bien solos o en colaboración con otros que no figuran entre los 10 primeros, el resultado es el siguiente: 41 citas de López del Ramo, 18 de Robledano Arillo, 16 de Laia Fox, 38 de Lluis Codina y 4 Antonio Ruiz.

Con respecto a cada uno de los artículos, el mayor número de citas lo tiene Lluis Codina con 21 para el texto "Bancos de imágenes y sonido, motores de indización en la www" (RE, 2011), seguido de Sánchez Vigil con 17 para "La fotografía como documento en el siglo XXI" (DC, 2001), Codina con 16 para "Entender los bancos de imágenes" (EP, 2011), López del Ramo con 15 para "Tratamiento fotográfico en webs periodísticas de la Universidad de Porto" (RE, 2015), y Laia Fox con 14 para "Patrimonio fotográfico de Cataluña en la red" (EP, 2011). 
Tabla VII. Materias generales y artículos.

\begin{tabular}{|l|c|l|c|}
\hline Materias & Art. & Materias & Art. \\
\hline Análisis y catalogación & 25 & Historia & 8 \\
\hline Archivos & 16 & Patrimonio & 3 \\
\hline Autoría & 10 & Prensa & 30 \\
\hline Bancos de imágenes & 7 & Recuperación & 8 \\
\hline Conservación & 4 & Redes sociales & 7 \\
\hline Docencia y Metodología & 6 & Revistas de fotografía & 6 \\
\hline Gestión (Derechos/Ética) & 8 & Tecnología & 24 \\
\hline Fondos y colecciones & 22 & Usos y aplicaciones & 27 \\
\hline
\end{tabular}

\subsection{Contenidos}

Con el fin de estructurar los contenidos, y una vez analizada la diversidad temática, se han establecido 16 apartados que contemplan el mayor número de temas tratados. Se han agrupado Docencia y Tecnología, al igual que Gestión (donde se incluyen Derechos y Ética, que solo cuentan con un artículo cada uno). En el apartado Tecnología se han incluido los temas de Digitalización (11 artículos), y en el de Usos y aplicaciones todos los asuntos generales más los especializados: Guerra (7), Publicidad (2), Medicina (1), Fotoperiodismo (3), Identidad (1) y Arquitectura (1). De manera específica se han abierto dos apartados por considerarlos de gran interés: Revistas de fotografía, por ser la materia principal (6) y Uso y aplicación en la prensa (30), por la cantidad de textos (tabla VII).

Los contenidos de los artículos hacen referencia a autores, instituciones privadas, universidades, y archivos, bibliotecas y museos. Aunque la prensa pertenezca al grupo de instituciones privadas, se ha considerado un apartado especial, al que se han sumado los trabajos en las redes, tanto por la cantidad como por la diversidad de estudios.

Autores (fotógrafos): Alfonso (Sánchez García y Sánchez Portela), Jorge Bosch Díaz, Mathew Brady, Robet, Capa, Robert. Adela Crooke, Manuel Durán Blázquez, Fernández Rivero (Colección), Dolores Gil, Eduardo y Francisco Hernández-Pacheco, Piedad Isla, Kaulak, Laurent, Mary Elen Mark, Francisco Martínez Gascón (Kautela), Bernardino Pardo, Emilio Pérez Piñero, Carlos Salvi Loscos, Gervasio Sánchez, Martín Santos Yubero, Miguel Trillo, y Joaquín Turina.

Universidades: Complutense de Madrid, República de Uruguay (Instituto de Cinematografía), Porto y Salamanca.

Archivos, Bibliotecas y Museos: Archivo de la Palabra y de la Imagen de Cartagena, Archivo de Santos de Maimona, Archivo General de la Administración del Estado (AGA), Archivo General de la Región de
Murcia, Municipal de Alcantarilla Municipal de San Javier, Regional de la Comunidad de Madrid, Subdirección General de Archivos Estatales (Censo-Guía de Archivos de España e Iberoamérica), Biblioteca Histórica Marqués de Valdecilla (UCM), Biblioteca Regional de Extremadura y Museo del Romanticismo.

Instituciones privadas: Diario $A b c$, agencia Efe, Ateneo de Madrid, Cecabank, Fundación Vicente Ferrer, Instituto de Valencia de Don Juan (2), Repsol y Unión de Informadores Gráficos de Prensa.

Prensa y redes: Avante (revista de fotografía), EI Diario de Palencia, El Diario Palentino, Flickr (2), Folha de Sao Paulo, Fotocinema (revista de foto y cine), Fotografía (revista), The Guardian, La Hormiga de Oro (revista), Instagram (4), Instantáneas (revista),

Tabla VIII. Palabras clave más utilizadas.

\begin{tabular}{|l|c|}
\hline Términos & Total \\
\hline Documentación fotográfica & 36 \\
\hline Archivos fotográficos & 30 \\
\hline Fondos y colecciones & 20 \\
\hline Análisis documental & 14 \\
\hline Prensa & 14 \\
\hline Gestión & 13 \\
\hline Digitalización & 11 \\
\hline Historia de la fotografía & 10 \\
\hline Fotoperiodismo & 10 \\
\hline Recuperación & 9 \\
\hline Patrimonio & 9 \\
\hline Bancos de imágenes & 8 \\
\hline Tratamiento documental & 7 \\
\hline Coleccionismo & 7 \\
\hline Prensa digital & 6 \\
\hline Metodología & 6 \\
\hline Imagen digital & 6 \\
\hline
\end{tabular}


Levante-EMV, El País (2), El Periódico, Unión fotográfica (revista), La Vanguardia y La Verdad.

Se han analizado además las palabras clave de los 146 artículos, con un resultado de 516 términos de los que sólo cuatro aparecen citados más de 15 veces (tabla V). La clave con mayor número de citas es Documentación fotográfica (36), seguida de Archivos fotográficos (30), Fondos y colecciones (20) y Análisis documental (14). De la cantidad y diversidad de términos se concluye la necesidad de una normalización con el fin de facilitar la recuperación. Es significativo que palabras clave de uso habitual en Documentación fotográfica aparezcan en muy pocos artículos, tales como banco de imágenes (8), indización (5), agencias (4), conservación (4), bases de datos (2), derechos (2) o tesauros (1). A partir de las 516 palabras clave indicadas por los autores, se han seleccionados aquellas más utilizadas (tabla VIII).

\section{DISCUSIÓN}

Cabe plantear algunas cuestiones que resultan significativas, la primera de ellas relacionada con estudios similares de carácter internacional en lo que se refiere a la producción en documentación fotográfica. En este sentido no se han localizado investigaciones, y en el caso español el trabajo que se presenta es el primer análisis sobre el tema. Algunos trabajos pueden considerarse aproximativos, si bien con enfoques diferentes a los que aquí se estudian, como por ejemplo La fotografía en Chile. Investigación y catastro de producción, difusión y levantamiento de información (Salgado, Canepa, 2010) realizado por el Consejo Nacional de la Cultura y las Artes de Chile y referido a la inserción de la fotografía en la sociedad, la industria y al mercado fotográfico; La documentación fotográfica en México: orígenes, evaluación y organización de los fondos, tesis doctoral de Ramos Fandiño (2009), que se ocupa del patrimonio y su tratamiento; los análisis de la producción en biblioteconomía, documentación y ciencias de la información en Brasil (Liberatore, 2015; Bufrem y otros, 2007), o el más amplio estudio sobre la producción científica archivística en la Red de Revistas Científicas de América Latina y el Caribe (Redalyc) durante el período 2001-2011 (Montilla Peña, 2012).

Un segundo aspecto que debe ser objeto de reflexión es el escaso número de investigaciones sobre la producción científica en las diferentes materias relacionadas con la Documentación, fundamentalmente las especializadas (documentación médica, jurídica, audiovisual, periodística, musical, etc.). En el caso de la documentación fotografía, el limitado número de artículos se concentra en una docena de autores, y su justificación podría encon- trarse en la falta de estudios oficiales de Grado y Máster para la formación de profesionales e investigadores, teniendo en cuenta que docencia e investigación están directamente relacionadas.

En cuanto al reducido número de citas totales y por artículo, consideramos que se debe precisamente al escaso número de especialistas. La falta de investigaciones no permite realizar una comparativa de la producción científica sobre materias especializadas en Documentación, si bien es excepción el "Estudio bibliométrico sobre la investigación en publicidad en España: temáticas, investigación, redes y centros de producción" (Baladrón Pazos y otros, 2017), cuyo resultado para un periodo inferior al que aquí se analiza (1980-2015) es de 1050 artículos de 1576 autores, frente a los 146 realizados por 161 sobre Fotografía, es decir una cantidad obviamente muy inferior en textos y autores que pone evidencia los distintos planos en que se encuentran ambas materias. Debemos puntualizar, sin embargo, que se han publicado trabajos sobre producción de tesis doctorales en comunicación (Jones y Bari, 2000), publicidad (Marcos Recio y otros, 2012), radio y televisión (Repiso y otros, (2011a y 2011b) y fotografía (Sánchez Vigil y otros, 2014, 2015), si bien la comparativa en ese campo excede los objetivos del artículo. Por otra parte, hacemos constar otros estudios que siendo tangentes vienen a completar los ya citados al tratarse de la visibilidad internacional de la producción científica iberoamericana en Biblioteconomía y Documentación en el periodo 1991-2000 (Moya Anegón y Herrero Solana, 2002), y sobre la autoría (Jiménez Contreras y Moya Anegón, 1997; Baladrón Pazos y otros, 2017).

Se abre también una vía de debate en lo que se refiere al habitual uso y aplicación de la fotografía en las diversas materias y disciplinas (arqueología, bellas artes, geología, medios de comunicación, ciencias en general, etc.), así como en la difusión de contenidos que, si bien es habitual gracias a su transversalidad, se margina en las investigaciones y por tanto no queda reflejado.

\section{CONCLUSIONES}

Una vez analizados los resultados, se concluye en primer lugar que los artículos sobre Fotografía en las revistas de Documentación son muy escasos $(2,18 \%$ del total) si tenemos en cuenta la ingente cantidad de documentos fotográficos que se conservan en archivos, bibliotecas, museos, bancos de imágenes, agencias y centros de documentación de las instituciones públicas y privadas, susceptibles de ser tratados y analizados para su uso y aplicación. Esta consideración global debe matizarse para poner en valor la aportación de determinadas revistas, 
como es el caso de Documentación de las Ciencias de la Información, con el 8,63\% de sus textos dedicados a la fotografía, Métodos de Información con el $6,64 \%$, Ibersid con el 5,11\% o Scire con el 3,01\%. Por el contrario, publicaciones como BiD, Revista Española de Documentación Científica y El Profesional de la Información (salvo el monográfico del año 2011) apenas reciben textos sobre la materia, con porcentajes inferiores a la media indicada.

La fotografía es, en términos generales, una materia de poca repercusión dentro del ámbito de la Documentación en la Universidad española. Aspectos como su tratamiento en forma y fondo, digitalización, conservación y restauración siguen teniendo muy poco peso dentro de la enseñanza superior. Pese a todo, el estudio constata que las universidades con asignaturas y grados afines a esta disciplina constituyen el principal núcleo generador de investigación y de artículos científicos, es el caso de la Complutense de Madrid (15 autores y 53 artículos), Granada (11 autores y 7 artículos), Carlos III de Madrid ( 7 autores y 8 artículos), Universidad de Barcelona (6 autores y 4 artículos), Autónoma Barcelona (5 autores y artículos), Pompeu Fabra ( 5 autores y 6 artículos) y Rey Juan Carlos (4 autores y 7 artículos).

En cuanto a los contenidos, además de las investigaciones relacionadas con las instituciones públicas responsables de la custodia de fondos y colecciones, hay una evidente tendencia al estudio de las redes y de los archivos y bancos de imágenes de prensa (Flickr, Instagram, $A b c$, agencia Efe, El Diario de Pa-

\section{REFERENCIAS}

Anef (2018). Asociación Nacional para la Enseñanza de la Fotografía. Orígenes y objetivos [en línea]. Disponible en: http://fotoanef.com. [Consulta: 20/12/2019].

Arquero Avilés, R. (2002). Autores más citados en publicaciones periódicas del área de Biblioteconomía y Documentación: España, 1975-1948. El Profesional de la Información, 11 (6), 436-441. https://doi. org/10.1076/epri.11.6.436.15464

Arquero Avilés, R. (2003). Evaluación de la colaboración científica en trabajos difundidos en publicaciones periódicas del área de Biblioteconomía y Documentación: España 1975-1984. Revista General de Información y Documentación, 13 (1), 247-259. https://doi. org/10.1076/epri.11.6.436.15464

Arquero Avilés, R.; Río Sadornil, J. L. de (2002). Trayectoria y estudio de producción de la revista Documentación de las Ciencias de la Información en su XXV aniversario (1976-2001). Documentación de las Ciencias de la Información, 25, 95-114.

Baladrón Pazos, A. J.; Correyero Ruiz, B. (2012). Futuro de las revistas científicas de comunicación en España. El Profesional de la Información, 21 (1), 34-42. https://doi.org/10.3145/epi.2012.ene.05 lencia, El Diario Palentino, Folha de Sao Paulo, The Guardian, La Hormiga de Oro, Levante-EMV, El País, El Periódico, La Vanguardia y La Verdad), así como de las revistas sobre fotografía: Avante, Fotocinema, Fotografía, Instantáneas y Unión fotográfica.

Desde el punto de vista de análisis documental, es importante señalar que algunos de los términos que figuran en los títulos de una parte considerable de los artículos no aparecen como palabras clave, lo que dificulta la recuperación de la información y aumenta su invisibilidad. Se sugiere, en consecuencia, que los editores de las revistas, a través de los sistemas de evaluación, fijen los criterios para ello, o bien incidan en este tema cuando las normas de evaluación son claras al respecto.

Así mismo, se considera necesario activar mecanismos para que los sistemas de búsqueda respondan a la demanda. Se ha detectado con frecuencia que ante términos con la misma base o prefijo ("foto" o "fotografía" son el ejemplo) la respuesta es diferente. Además, algunas revistas no permiten la búsqueda por palabras clave o por el título, por lo que ha sido imprescindible revisar todos y cada uno de los números publicados.

Finalmente, en referencia a la cuantía de textos, es obvia la necesidad de dinamizar la investigación en documentación fotográfica, con el fin de aumentar la producción científica, prestando mayor atención a su estudio. Entendemos que una forma de hacerlo sería la convocatoria periódica de monográficos por los agentes implicados.

Baladrón Pazos, A. J.; Manchado Pérez, B.; Correyero Ruiz, B. (2017). Estudio bibliométrico sobre la investigación en publicidad en España: temáticas, investigación, redes y centros de producción. Revista Española de Documentación de Científica, 40 (2). https://doi. org/10.3989/redc.2017.2.1411

Bufrem, Leilah S. y otros (2007). Produção científica em ciência da informação: análise temática em artigos de revistas brasileiras, Perspectivas em Ciência da Informação, vol.12, 1, pp. 38-49. https://doi.org/10.1590/ S1413-99362007000100004

Caffarel Serra, C.; Ortega Mohedano, F.; Gaitán Moya, J.A. (2017). Investigación en Comunicación en las Universidades españolas en el periodo 2007-2014. El Profesional de la Información, 26 (2), 2018-227. https:// doi.org/10.3145/epi.2017.mar.08

Cerdán Medina, J. C.; Ortega Priego, J. L.; Rodríguez Yunta, L. (2015). Caracterización de las revistas Españolas de Biblioteconomía y Documentación: Producción científica y perfil temático en el periodo 2004-2013. Madrid: CSIC-ISOC

Delgado López-Cózar, E. (2001). Las revistas españolas de Ciencias de la Documentación: productos manifiestamente mejorables. El Profesional de la Información, 10 (12), 46-56. https://doi.org/10.1076/epri.10.12.46.6548 
Ferrer Sapena, A.; Sánchez Pérez, E. A.; Benavent, A. y Peset, F. (2016). Cómo analizar el impacto de los datos de investigación con métricas: modelos y servicios, $E L$ profesional de la Información, 25 (4), 632-641. https:// doi.org/10.3145/epi.2016.jul.13

Gorraiz, J. (2018). Los mil y un reflejo de las publicaciones en el laberinto de espejos de las nuevas métricas. EL profesional de la Información, 27 (2), 231-236. https:// doi.org/10.3145/epi.2018.mar.01

Grupo Scimago (2005). Producción española con visibilidad internacional (ISI-WOS) en biblioteconomía y documentación (I). El Profesional de la Información, 14 (6), 459461.

Grupo Scimago (2006). Producción española con visibilidad internacional (ISI-WOS) en biblioteconomía y documentación (II). El Profesional de la Información, 15 (1), 3436. https://doi.org/10.3145/epi.2005.nov.08

Guallar, J. (2003). Métodes i tècniques de recerca en els articles de documentació periodística a Espanya (19972002), BiD, 11. http://bid.ub.edu/11gualla.htm

Guallar, J.; Ferran-Ferrer, N.; Abadal, E. y Server, A. (2017). Revistas científicas de información y documentación: Análisis temático y metodológico. El Profesional de la Información, 26 (5), 947-960. https://doi.org/10.3145/ epi.2017.sep.16

Jiménez Contreras, E.; Delgado López-Cózar, E.; Ruiz Pérez, R. (2006). Producción española en biblioteconomía y documentación con visibilidad internacional a través del Web of Science (1995-2004). El Profesional de la Información, 15 (5), 373-383. https://doi.org/10.3145/ epi.2006.sep.06

Jiménez Contreras, E.; Moya Anegón, F. (1997). Análisis de la autoría en revistas españolas de biblioteconomía y documentación. Revista Española de Documentación Científica, 20 (3), 252-266. https://doi.org/10.3989/ redc. 2007.v30.i3.387

Jiménez Hidalgo, S. (2007). Análisis de la autoría en la Revista Española de Documentación Científica (19972005). Revista Española de Documentación Científica, 30 (3), 305-322.

Jones, D. E.; Barí I Queralt, B. (2000). Investigación sobre Comunicación en España. Aproximación bibliométrica a las tesis doctorales (1926-1998). Barcelona: ComCat, p. 152.

Liberatore, G. H. (2015). Análisis bibliométrico de la producción científica en bibliotecología y ciencia de la información en Brasil en el periodo 2000-2011: Estudio de cuatro revistas nacionales de la disciplina [Tesis Doctoral]. Madrid: Universidad Carlos III

López López, P, y otros (2001). Estudio bibliométrico y de calidad de la revista General de Información y Documentación (1991-2000). Revista General de Información y Documentación, 11 (1), 11-32. https://doi.org/10.1076/ epri.11.1.46.9061

López Yepes, J.; Fernández Bajón, M. T.; Prat Sedeño, J. (2005). La investigación española en documentación informativa a examen en Ibersid 2004. Estado de la cuestión con especial referencia a las tesis doctorales (19762004). El Profesional de la Información, 14 (1), 50-58. https://doi.org/10.3145/epi.2005.feb.08

Marcos Recio, J. C.; Martínez Pestaña, M. J.; Blasco López, M. F. (2012). Producción y dirección de tesis doctorales sobre publicidad en la universidad española (1971-
2010). Revista Española de Documentación Científica, 35 (3), 433-452. https://doi.org/10.3989/redc.2012.3.890

Ministerio de Cultura (2015). Plan Nacional para la conservación del patrimonio fotográfico. Madrid: IPCE.

Montilla Peña, Leomar José (2012). Análisis bibliométrico sobre la producción científica archivística en la Red de Revistas Científicas de América Latina y el Caribe (Redalyc) durante el período 2001-2011. Biblos, 2012, 48, 1-11. https://doi.org/10.5195/BIBLIOS.2012.65

Moya Anegón, F.; Herrero Solana, V. (2002). Visibilidad internacional de la producción científica iberoamericana en biblioteconomía y documentación (1991-2000). Ciência da Informação, 31 (3), 54-65. https://doi.org/10.1590/ S0100-19652002000300006

Olivera Zaldua, M.; Sánchez Vigil, J. M.; Marcos Recio, J. C. (2016). Análisis de las tesis doctorales sobre fotografía en la universidad española (enero de 2013-marzo de 2016). Ibersid, 10 (2), 13-20.

Osca LLuch, J.; Mateo Marquina, E. (2003). Difusión de las revistas españolas de ciencias sociales y humanidades. Revista General de Información y Documentación, 13 (1), 115-132.

Ramos Fandiño, P. (2009). La documentación fotográfica en méxico: orígenes, evaluación y organización de los fondos. Tesis doctoral. Madrid: Universidad Complutense.

Repiso, R.; Torres, D.; Delgado, E. (2011a): Análisis bibliométrico y de redes sociales en tesis doctorales españolas sobre televisión (1976-2007). Comunicar, vol. 37 (XIX), 151-159.

Repiso, R.; Torres, D.; Delgado, E. (2011b). Análisis de la investigación sobre Radio en España: una aproximación a través del análisis bibliométrico y de Redes Sociales de las tesis doctorales defendidas en España entre 19762008. Estudios sobre el mensaje periodístico, vol. 17 (2), 417-429. https://doi.org/10.5209/rev_ESMP.2011. v17.n2.38123

Ríos Hilario, A. B. (2001). Diez años de investigación en la Revista Española de Documentación Científica (19891999). Revista Española de Documentación Científica, 24 (4), 433-439. https://doi.org/10.3989/redc.2001. v24.i4.70

Salgado Tello, S. ; Canepa Castro, E. (2010). La fotografía en Chile. Investigación y catastro de producción, difusión y levantamiento de información. Santiago de Chile: Centro Nacional del Patrimonio Fotográfico. Área de Fotografía del Departamento de Creación Artística, Consejo Nacional de la Cultura de las Artes.

Salvador Bruna, J. (2007). Top ten. Diez años de investigación española en biblioteconomía y documentación (1996-2006). Ranking e Índice $\mathrm{H}$ de los 10 autores en activo más citados. Revista General de Información y Documentación, 17 (2), 159-182.

Sánchez Vigil, J. M.; Marcos Recio, J. C.; Olivera Zaldua, M. (2014). Tesis doctorales sobre fotografía en la universidad española: análisis de la producción científica (19762012). Revista Española de Documentación Científica, 37 (1). https://doi.org/10.3989/redc.2014.1.1073

Sánchez Vigil, J. M.; Marcos Recio, J. C.; Olivera Zaldua, M. (2015). Producción científica sobre comunicación y medios en las revistas de documentación (2000-2014). Revista Española de Documentación Científica, 38 (4). https://doi.org/10.3989/redc.2015.4.1257 


\section{ANEXO 1. AUTORES Y REVISTAS}

Esquema: Apellido, N.: Revista Año/Volumen

Abreviaturas: AN: Anales; BA: Boletín de Anabad; BD: Biblioteconomía i Documentació; CD: Cuadernos de Documentación Multimedia; DC: Documentación de las Ciencias de la Información; EP: El Profesional de la Información; IB: Ibersid; MI: Métodos de Información; RE: Revista Española de Documentación Científica; RG: Revista General de Información y Documentación; SC: Scire.

Acal, I.; RG 2015/25-2

Aguillo, I. F.: EP 2008/17-6

Agustin, C.: RG 2018/28-2, 2019/29-2

Alberich, J.: EP 2018/27-1, 2019/28-1

Alfaro, H. G.: RV 2019/29-1

Alonso, J.: EP 2011/20-4; ME 2014/5

Alvoz, E.: DC 2017/40

Arango, R.: IB 2007/1

Ardanuy, J.: DC 2013/36

Arias, R.: RG 2014/24-1

Belda, R.: IB 2007/1

Berdaguer, X.: EP 2000/9-3

Bizello, M. L.: IB 2009/3

Blanco, J. C.: EP2009/18-3

Blázquez, M.: SC 2012/18-2

Borsetti, S. A.: RG 2016/26-2

Brugarolas, C. M.: SC 2002/8-2

Caldera, J.: MI 2014/5

Cancela, G.: EP 2014/23-3

Cantón Correa, F.J.: EP 2019/28-1

Cascón J.: EP 2018/27-1, 2019/28-4

Castellano, M. Á.: EP 2014/23-5

Castillo, S.: EP 2011/20-4

Castillo Fernández, J.: MI 2014/5

Castro, L. de: DC 2019/42

Cirio, A. L.: BA 2011/61-2

Clavería, R.: DC 2015/38

Clavero Galofré, M.: RG 2018/29-2

Clavero Suero, F.: DC 2017/40

Codina, L.: EP: 2007/16-5, 2011/20-4;

RE 2001/24-3;

Cordón, J. A.: IB 2007/1

Dal' Evedove, P. R.: SC 2010/16-1, 2010/16-2

Deltell, L.: EP: 2017/26-6

Díaz, M.: EP 2017/26-1

Doménech, H.: EP 2018/27-5

Domínguez, J. J.: IB 2007/1

Dorado, P.: DC 2013/36

Elliott, A. G.: IB 2013/7

Fernández, M.: BA 2011/61-2

Fernández Fuentes, B.: DC 2005/28; SC 2005/11-1

Fernández Rivero, J. A.: ME 2014/5

Fijita, M. S. L.: SC 2010/1

Firderes, J. E.: DC 2016/39

Fontanela, L.: 2011/20-4

Fox, L.: EP 2011/20-4; DC 2013/36; ME2014/5
Freixa, P.: EP 2011/20-4

Fresnedillo, S.: MI 2014/5

Fuentes, M. E: IT 2002/37

García, M. T.: MI 2014/5

Garrido, C.: BA 2018/3

Garrote, E.: EP 2014/23-6

Gil Almela, M. R.: BA 2016/66-3

Gil Leiva, I.: AD 2002/5

Gómez Díaz, R.: IB 2007/1

Gómez Egea, J. M.: IB 2007/1

Gómez Isla, J.: IB 2007/1

Gonçalves J.: EP 2014/23-3

González Coll, R.: DC 2016/39

González Quesada, A.: IT 2002/37

González Rodríguez, M.: EP 2012/21-1

González San Juan, J. L. IB 2015/9

Granados, A.: EP 2019/28-3

Gutiérrez, M. A.: AD 2011/14-2

Gutiérrez Chiñas, A.: DC 2011/34

Heras, B. de las: RG 2014/24-2

Herrera Morillas, J. L.: BD 2011/27

Herrera Viedma, E.: EP 2019/28-4

Huertas, A.: EP 2015/24-1

Humanes, M. L.: SC 2016/22-2

Jiménez Fernández, P.: IT 2018/64

Jiménez López, À.: IT 2002/37

León, J A.: MI 2014/5

Lima, M. de L.: SC 2016/22-1

Llovet, C.: EP 2017/26-1

Lluch, L.: SC 2002/8-2

Lopes, M. S.: IB 2009/3

López Ávila, M. B.: RE 2015/38-2

López del Ramo, J.: IB 2008/1; EP 2010/19-5; RE 2015/25-5, 2017/40-2; SC 2016/22-5

López Herrera, A. G.: EP 2019/28-4

López Rabadán, P.: EP 2018/27-5

Losada Fernández, C.: IT 2018/64

Madio, T. C. C.: IB 2009/3, 2010/4, 2013/7, 2016/10-2

Marcos, M. C.: EP2014/23-3

Marcos Recio, J. C.: EP 2010/19-1; IB 2007/1, 2013/7, 2014/8, 2015/9, 2016/10-2, 2017/11-2; RE 2014/37$1 ; 2017 / 40-2,2018 / 41-3$; SC 2005/1-2, 2005/11-1

Martín, M.: SC2002/8-2

Martínez de Pisón, E.: EP 2014/23-3

Martínez de Vega, C.: RG 2016/26-2

McGowan, N.: EP 2017/26-6

Míguez, M. I.: EP 2015/24-1 
Milena, S.: RG 2016/26-2

Millán, F.: BA 2015/65-3

Mora, V.: RG 2014/24-2

Moreno, R. A.: AD 2011/14-2

Moreno Pelayo, V.: EP2019/28-3;

RE 2016/39-2

Muñoz Castaño, J. E.: EP 2001/10-3

Navarro, D.: EP 2017/26-6

Navas, J.: EP: 2011/20-4

Nuño Moral, M. V.: RG 2019/29-1

Oliveira, I.: SC: 2010/16-2

Olivera, M.: BA 2017/67-1; AD 2012/15-2, 2015/18-1; BD 2018/41; CD 2016/27-2; DC 2010/33, 2011/34, 2011/36, 2014/37 2017/40; EP 2010/19-1; IB 2013/7, 2014/8, 2015/9,2016/10-2, 2017/11-2; MI 2014/5; RE 2014/37-1, 2018/41-3

Ortega, J. L.: EP 2008/17-6

Ortiz, L.: EP 2014/23-3

Palao, J. M.: DC 2016/39

Palma, M. del V.: RE 2001/24-3

Palomares, R.: SC 2016/22-2

Parras, A.: RG 2010/20; DC 2012/35, 2013/36

Pastor, J. A.: EP 2011/20-4

Paz, L. E.: MI 2017/8-14

Pedreira, G.: BA 2015/65-3

Perdices, L.: EP 2011/20-4, 2014/32-5

Pereira, J. M.: EP 2013/22-3; RE 2016/39-2

Pérez Almagro, M. C.: AD 2017/20-2

Pérez Álvarez, S.: DC 2003/26, 2006/29

Pérez Borges, A.: RE 2011/34-2

Periago, L.: EP 2001/10/10

Perianes, A.: EP 2011/20-4, 2014/23-5

Potalés, M.: EP 2017/26-5

Prat, J.: SC 2006/12-2

Pupim, E. K.: IB 2009/3, 2010/4

Quevedo, R.: EP 2017/26-5

Ramírez, M.: EP 2014/23-5

Ramos, G. P.: DC 2011/34

Rebelo, M. de Sa: AD 2011/14-2

Río, M. J.: MI 2014/5
Robledano, J.: EP 2013/22-3, 2017/26-6, 2019/28-3; RE 2011/34-3, 2016/39-2

Rodero, A.: EP 2009/18-3

Rodríguez Cela, J: DC 2012/35; RG 2010/20

Rodríguez Vaamonde, S.: EP 2012/21-1, 2014/23-6

Ros Martín, M.: EP 2009/18-3

Ros Sempere, M.: CD 2009/20

Rosa, A. de la: EP 2003/12-5

Rovira, C.: EP 2014/23-3

Ruiz Ibáñez, P.: EP 2012/21-1

Ruiz Rodríguez, A. Á.: EP 2011/20-4, 2018/27-1, 2019/28-4

Runiano, P.: RG 2019/29-1

Salvador, A.: BA 2017/67-1; BD 2018/41; CD 2016/27-2; RE 2016/39-2

Sánchez Hernández, M.F.: SC 2007/13-1

Sánchez Vigil, J. M.: AD 2012/15-2, 2015/18-1, 2016/192, 2018/21-2; BA 2005/55-1-2, 2007/57-2, 2017/671 BD 2018/41; IB 2007/1, 2013/7, 2014/8, 2015/9; 2016/10-2, 2017/11-2; CD 2016/27-2; DC 2001/24, 2005/28, 2013/36, 2014/37, 2017/40; EP 2010/19-1

(2), 2011/20-4; MI 2014/5; RE 2014/37-1, 2016/39-2, 2018/41-3; RG 2016/26-1; SC 2005/11-1, 2005/11-2

Sansoni, I. M.: IB 2016/10-2

Saorín, T.: EP 2011/20-4

Senso, J. A. EP 2003/12-5

Setó, D.: EP 2015/24-1

Silva, I. de O. S.: IB 2009/3; SC 2010/1

Sol, B. del: MI 2017/8-14

Soler, R.: IT 2003/33

Spotti, M.: SC 2010/16-2

Suárez, J. P.: EP 2014/23-5

Tinoco, J.: IT 2015/64

Tomás. S.: RG 2018/28-2

Torre, I.: EP 2014/23-6

Torregrosa, J. F.: DC 2010/33

Troitiño, S. M.: IB 2016/10-2

Vicentini, M. J.: RG 2016/26-2

Villegas, R.: IB 2007/1; SC 2005/11-2

Vivas, A.: RG 2019/29-1 